\title{
A Linearized Relaxing Algorithm for the Specific Nonlinear Optimization Problem
}

\author{
Mio Horai, ${ }^{1,2}$ Hideo Kobayashi, ${ }^{1}$ and Takashi G. Nitta ${ }^{3}$ \\ ${ }^{1}$ Faculty of Engineering, Graduate School of Engineering, Mie University, Kurimamachiyamachi, Tsu 514-8507, Japan \\ ${ }^{2}$ Ise Gakuen High School, Kurosecho, Ise 516-0018, Japan \\ ${ }^{3}$ Department of Mathematics, Mie University, Kurimamachiyamachi, Tsu 514-8507, Japan
}

Correspondence should be addressed to Mio Horai; mio@com.elec.mie-u.ac.jp

Received 27 December 2015; Accepted 26 April 2016

Academic Editor: Lucas Jódar

Copyright (C) 2016 Mio Horai et al. This is an open access article distributed under the Creative Commons Attribution License, which permits unrestricted use, distribution, and reproduction in any medium, provided the original work is properly cited.

We propose a new method for the specific nonlinear and nonconvex global optimization problem by using a linear relaxation technique. To simplify the specific nonlinear and nonconvex optimization problem, we transform the problem to the lower linear relaxation form, and we solve the linear relaxation optimization problem by the Branch and Bound Algorithm. Under some reasonable assumptions, the global convergence of the algorithm is certified for the problem. Numerical results show that this method is more efficient than the previous methods.

\section{Introduction}

Optimization problems appeared in many subjects [1-3], for example, technology [4-7] and economy [8-10]. There is a long history of creating the method for solving the problem [11-14]. We consider the following certain nonlinear optimization problem on the set $X:=\left\{x \in \mathbf{R}^{N} \mid 0<\underline{x}_{i} \leq\right.$ $\left.x_{i} \leq \bar{x}_{i}<\infty(i=1,2, \ldots, N)\right\} \subset \mathbf{R}^{N}$.

Let $T_{j}^{a}, T_{j}^{b}, T_{k j}^{c}, T_{k j}^{d}$ be natural numbers, let $\beta_{j t}^{a}, \beta_{j t}^{b}, \beta_{k j t}^{c}$, $\beta_{k j t}^{d}$ be nonzero real constants, and let $\gamma_{j t i}^{a}, \gamma_{j t i}^{b}, \gamma_{k j t i}^{c}, \gamma_{k j t i}^{d}$ be real constants. Then we put the four kinds of functions on $X$ :

$$
\begin{gathered}
a_{j}(x):=\sum_{t=1}^{T_{j}^{a}} \beta_{j t}^{a} \prod_{i=1}^{N} x_{i}^{\gamma_{j t i}^{a},} \\
b_{j}(x):=\sum_{t=1}^{T_{j}^{b}} \beta_{j t}^{b} \prod_{i=1}^{N} x_{i}^{\gamma_{j t i}^{b},} \\
c_{k^{\prime} j}(x):=\sum_{t=1}^{T_{k j}^{c}} \beta_{k j t}^{c} \prod_{i=1}^{N} x_{i}^{\gamma_{k j t i}^{c},}
\end{gathered}
$$

$$
\begin{aligned}
d_{k j}(x) & :=\sum_{t=1}^{T_{k j}^{d}} \beta_{k j t}^{d} \prod_{i=1}^{N} x_{i}^{\gamma_{k j t i}^{d}}, \\
(j & \left.=1,2, \ldots, P, \dot{j}=1,2, \ldots, P_{k}, k=1,2, \ldots, M\right) .
\end{aligned}
$$

Let $h_{j}, h_{k^{\prime}}$ be two secondly differentiable functions $\mathbf{R} \mapsto \mathbf{R}$ satisfying the following conditions:

$$
\begin{array}{r}
h_{j}^{\prime}>0 \\
\text { or } h_{j}^{\prime}<0, \\
h_{k^{\prime} j}^{\prime}>0 \\
\text { or } h_{k^{\prime}}^{\prime}<0, \\
\left\{h_{j}\right\}^{\prime \prime}>0 \\
\text { or }\left\{h_{j}\right\}^{\prime \prime}<0, \\
\left\{h_{k^{\prime}}\right\}^{\prime \prime}>0 \\
\text { or }\left\{h_{k_{j}}\right\}^{\prime \prime}<0 .
\end{array}
$$


We consider the following nonlinear optimization problem $(P)$ on $X$ :

$$
\begin{array}{ll}
\min & f_{0}(x)=\sum_{j=1}^{P} h_{j}\left(\frac{b_{j}(x)}{a_{j}(x)}\right) \\
\text { s.t. } & f_{k}(x)=\sum_{j=1}^{P_{k}} h_{k^{\prime} j}\left(\frac{d_{k^{\prime} j}(x)}{c_{k^{\prime} j}(x)}\right) \leq 0 \\
& \left({ }^{\prime} j=1, \ldots, P_{k}, k=1, \ldots, M\right) .
\end{array}
$$

We propose a specific nonlinear and nonconvex optimization technique for $(P)$. It is generalized by Jiao et al., 2013 [4].

In the previous our work [15], we treat the same problem $(P)$ applying Pei-Ping and Gui-Xia's [5]. The method needs to add the new valuables and takes a long time to solve the optimal problem.

Jiao et al. propose the technique which does not launch new ones for the following problems:

$$
\begin{array}{ll}
\min & h_{0}(x)=\sum_{j=1}^{P} \frac{b_{j}(x)}{a_{j}(x)} \\
\text { s.t. } & h_{k}(x)=\sum_{j=1}^{P_{k}} c_{k j}(x) \\
& \leq 0\left({ }^{\prime} j=1, \ldots, P_{k}, k=1, \ldots, M\right), \\
& x \in X .
\end{array}
$$

We generalize the problem $\left(P^{*}\right)$ to $(P)$ and use Hongwei's idea $[4,16]$ to solve the problem $(P)$; that is, we propose the new method by generalizing Hongwei's method.

Firstly, we transform $(P)$ to linear relaxation problem of it. Secondly, we obtain the approximate value by Simplex method and Branch and Bound Algorithm [17, 18]. For advance preparation of the linearization, we transform the valuables $x_{i}=\exp \left(y_{i}\right)(i=1,2, \ldots, N)$. Let $\underline{x}_{i}:=\exp \left(y_{i}\right)$, $\bar{x}_{i}:=\exp \left(\bar{y}_{i}\right)$, and $Y:=\left\{y \in \mathbf{R}^{N} \mid \underline{y}_{i} \leq y_{i} \leq \bar{y}_{i}<\infty \overline{(i}=\right.$ $1,2, \ldots, N)\}$.

We denote

$$
\begin{aligned}
a_{j}(y) & :=\sum_{t=1}^{T_{j}^{a}} \beta_{j t}^{a} \exp \left(\sum_{i=1}^{N} \gamma_{j t i}^{a} y_{i}\right), \\
b_{j}(y):= & \sum_{t=1}^{T_{j}^{b}} \beta_{j t}^{b} \exp \left(\sum_{i=1}^{N} \gamma_{j t i}^{b} y_{i}\right), \\
c_{k^{\prime} j}(y):= & \sum_{t=1}^{T_{k j}^{c}} \beta_{k^{\prime} j t}^{c} \exp \left(\sum_{i=1}^{N} \gamma_{k j t i}^{c} y_{i}\right), \\
d_{k^{\prime} j}(y):= & \sum_{t=1}^{T_{k j}^{d}} \beta_{k^{\prime} j t}^{d} \exp \left(\sum_{i=1}^{N} \gamma_{k j t i}^{d} y_{i}\right) \\
(j= & \left.1,2, \ldots, P, \quad j=1,2, \ldots, P_{k}, k=1,2, \ldots, M\right) .
\end{aligned}
$$

Accordingly, we obtain the equivalence problem of $(P)$ :

$$
\begin{array}{ll}
\min & f_{0}(y)=\sum_{j=1}^{P} h_{j}\left(\frac{b_{j}(y)}{a_{j}(y)}\right) \\
\text { s.t. } & f_{k}(y)=\sum_{j=1}^{P_{k}} h_{k^{\prime} j}\left(\frac{d_{k^{\prime}}(y)}{c_{k^{\prime}}(y)}\right) \\
& \leq 0\left({ }^{\prime} j=1, \ldots, P_{k}, k=1, \ldots, M\right) \\
& y \in Y .
\end{array}
$$

As the function "exp" is convex function, we find the lower and upper bounded linearized function of it.

In Section 2, we show how to linearize the original problem $(P)$. In Section 3, we present our method by using the Branch and Bound Algorithm. In Section 4, we prove the convergence of the algorithm. In Section 5, we treat numerical experiments.

\section{Linear Relaxation Programing}

In this section, we show how to transform $(P 0)$ to the linear relaxation problem.

We define

$$
Y_{j t}^{a}:=\sum_{i=1}^{N} \gamma_{j t i}^{a} y_{i}
$$

Corresponding to the transformation of coordinates, the domain is changed from $y$ to $Y$, as follows:

$$
\begin{aligned}
& \underline{Y}_{j t}^{a}:=\sum_{i=1}^{N} \min \left\{\gamma_{j t i}^{a} \underline{y}_{i}, \gamma_{j t i}^{a} \bar{y}_{i}\right\}, \\
& \bar{Y}_{j t}^{a}:=\sum_{i=1}^{N} \max \left\{\gamma_{j t i}^{a} \underline{y}_{i}, \gamma_{j t i}^{a} \bar{y}_{i}\right\} .
\end{aligned}
$$

Since all $\beta_{j t}^{a} \exp \left(Y_{j t}^{a}\right)$ are convex, there exist the lower and upper bounded linear functions for them. We denote these functions by $L_{\beta_{j t}^{a}}^{l}\left(Y_{j t}^{a}\right)$ and $L_{\beta_{j t}^{a}}^{u}\left(Y_{j t}^{a}\right)$.

When $\beta_{j t}^{a}$ is negative, we can define the lower linearized function $L_{\beta_{j t}^{a}}^{l}\left(Y_{j t}^{a}\right)$ :

$$
\begin{aligned}
L_{\beta_{j t}^{a}}^{l}\left(Y_{j t}^{a}\right):= & \frac{\beta_{j t}^{a} \exp \left(\bar{Y}_{j t}^{a}\right)-\beta_{j t}^{a} \exp \left(\underline{Y}_{j t}^{a}\right)}{\bar{Y}_{j t}^{a}-\underline{Y}_{j t}^{a}}\left(Y_{j t}^{a}-\underline{Y}_{j t}^{a}\right) \\
& +\beta_{j t}^{a} \exp \left(\underline{Y}_{j t}^{a}\right) .
\end{aligned}
$$

Since each $\beta_{j t}^{a} \exp \left(Y_{j t}^{a}\right)$ is continuous and differentiable on $\left[\underline{Y}_{j t}^{a}, \bar{Y}_{j t}^{a}\right]$, there exists $c_{j t}^{a} \in\left(\underline{Y}_{j t}^{a}, \bar{Y}_{j t}^{a}\right)$ such that

$$
\left\{\beta_{j t}^{a} \exp \right\}^{\prime}\left(c_{j t}^{a}\right)=\frac{\beta_{j t}^{a} \exp \left(\bar{Y}_{j t}^{a}\right)-\beta_{j t}^{a} \exp \left(\underline{Y}_{j t}^{a}\right)}{\bar{Y}_{j t}^{a}-\underline{Y}_{j t}^{a}}
$$

by the mean value theorem. 
Since $\beta_{j t}^{a} \exp \left(Y_{j t}^{a}\right)$ is monotonic function on $\left[\underline{Y}_{j t}^{a}, \bar{Y}_{j t}^{a}\right]$, there exists the inverse function of $\left\{\beta_{j t}^{a} \exp \right\}^{\prime}\left(Y_{j t}^{a}\right)$. Hence $c_{j t}^{a}$ is uniquely given such that

$$
c_{j t}^{a}=\left\{\beta_{j t}^{a} \exp \right\}^{\prime^{-1}}\left(\frac{\beta_{j t}^{a} \exp \left(\bar{Y}_{j t}^{a}\right)-\beta_{j t}^{a} \exp \left(\underline{Y}_{j t}^{a}\right)}{\bar{Y}_{j t}^{a}-\underline{Y}_{j t}^{a}}\right) .
$$

When $\beta_{j t}^{a}$ is positive, we define $L_{\beta_{j t}^{a}}^{l}\left(Y_{j t}^{a}\right)$ :

$$
\begin{aligned}
L_{\beta_{j t}^{a}}^{l}\left(Y_{j t}^{a}\right):= & \frac{\beta_{j t}^{a} \exp \left(\bar{Y}_{j t}^{a}\right)-\beta_{j t}^{a} \exp \left(\underline{Y}_{j t}^{a}\right)}{\bar{Y}_{j t}^{a}-\underline{Y}_{j t}^{a}}\left(Y_{j t}^{a}-c_{j t}^{a}\right) \\
& +\beta_{j t}^{a} \exp \left(c_{j t}^{a}\right) .
\end{aligned}
$$

For the upper linearized function of $\beta_{j t}^{a} \exp \left(Y_{j t}^{a}\right)$, we define $L_{\beta_{j t}^{u}}^{u}\left(Y_{j t}^{a}\right)$ as follows.

When $\beta_{j t}^{a}$ is negative, we define

$$
\begin{aligned}
L_{\beta_{j t}^{a}}^{u}\left(Y_{j t}^{a}\right):= & \frac{\beta_{j t}^{a} \exp \left(\bar{Y}_{j t}^{a}\right)-\beta_{j t}^{a} \exp \left(\underline{Y}_{j t}^{a}\right)}{\bar{Y}_{j t}^{a}-\underline{Y}_{j t}^{a}}\left(Y_{j t}^{a}-c_{j t}^{a}\right) \\
& +\beta_{j t}^{a} \exp \left(c_{j t}^{a}\right) .
\end{aligned}
$$

When $\beta_{j t}^{a}$ is positive, we define $L_{\beta_{j t}^{a}}^{u}\left(Y_{j t}^{a}\right)$ :

$$
\begin{aligned}
L_{\beta_{j t}^{a}}^{u}\left(Y_{j t}^{a}\right):= & \frac{\beta_{j t}^{a} \exp \left(\bar{Y}_{j t}^{a}\right)-\beta_{j t}^{a} \exp \left(\underline{Y}_{j t}^{a}\right)}{\bar{Y}_{j t}^{a}-\underline{Y}_{j t}^{a}}\left(Y_{j t}^{a}-\underline{Y}_{j t}^{a}\right) \\
& +\beta_{j t}^{a} \exp \left(\underline{Y}_{j t}^{a}\right) .
\end{aligned}
$$

As the above definitions, we have the lower and upper linearized functions of $a_{j}(y)$; that is,

$$
\begin{aligned}
& l_{a_{j}}^{l}(y):=\sum_{t=1}^{T_{j}^{a}} L_{\beta_{j t}^{a}}^{l}\left(Y_{j t}^{a}\right), \\
& l_{a_{j}}^{u}(y):=\sum_{t=1}^{T_{j}^{a}} L_{\beta_{j t}^{a}}^{u}\left(Y_{j t}^{a}\right),
\end{aligned}
$$

and $l_{b_{j}}^{l}(y), l_{b_{j}}^{u}(y), l_{c_{k_{j}}}^{l}(y), l_{c_{k j}}^{u}(y), l_{d_{k j}}^{l}(y)$, and $l_{d_{k^{j}}}^{u}(y)$ are also defined for $b_{j}(y), c_{k^{\prime} j}(y)$, and $d_{k^{\prime}}(y)$ as the same method. Moreover, we can assume $l_{a_{j}}^{l}(y)>0, l_{b_{j}}^{l}(y)>0, l_{c_{k j}}^{l}(y)>0$, and $l_{d_{k^{\prime}}}^{l}(y)>0$ by adding some constraints.

Now, we define the new valuables $Y_{a b}^{j} \in\left[\min l_{b_{j}}^{l}(y) /\right.$ $\left.\max l_{a_{j}}^{u}(y), \max l_{b_{j}}^{u}(y) / \min l_{a_{j}}^{l}(y)\right]$ and $Y_{c d}^{k^{\prime j}} \in\left[\min l_{d_{k j}}^{l}(y) /\right.$ $\left.\max l_{c_{k j}}^{u}(y), \max l_{d_{k j}}^{u}(y) / \min l_{c_{k^{k}}}^{l}(y)\right]$. Let us consider the lower linearized functions $l_{h_{j}}, l_{h_{k j}}$ for $h_{j}, h_{k^{\prime} j}$. We suppose $l_{h_{j}}$ as follows.
Case $1\left(\left\{h_{j}\right\}^{\prime}>0\right)$. In the case, we put the valuable $Y_{a b}^{j} \in$ $\left[\underline{Y}_{a b}^{j}, \bar{Y}_{a b}^{j}\right]$; that is, $\underline{Y}_{a b}^{j}:=\min l_{b_{j}}^{l}(y) / \max l_{a_{j}}^{u}(y), \bar{Y}_{a b}^{j}:=$ $\max l_{b_{j}}^{l}(y) / \max l_{a_{j}}^{u}(y)$.

$$
\text { As }\left\{h_{j}\right\}^{\prime}>0, h_{j}\left(l_{b_{j}}^{l}(y) / l_{a_{j}}^{u}(y)\right) \leq h_{j}\left(b_{j}(y) / a_{j}(y)\right) \text {. }
$$

When $\left\{h_{j}\right\}^{\prime \prime}<0$, we define the lower linearized functions of $h_{j}$ as follows:

$$
\begin{aligned}
l_{h_{j}}\left(Y_{a b}^{j}\right):= & \frac{h_{j}\left(\bar{Y}_{a b}^{j}\right)-h_{j}\left(\underline{Y}_{a b}^{j}\right)}{\bar{Y}_{a b}^{j}-\underline{Y}_{a b}^{j}}\left(Y_{a b}^{j}-\underline{Y}_{a b}^{j}\right) \\
& +h_{j}\left(\underline{Y}_{a b}^{j}\right) .
\end{aligned}
$$

Incidentally, as $h_{j}\left(Y_{a b}^{j}\right)$ is continuous and differentiable on $\left[\underline{Y}_{a b}^{j}, \bar{Y}_{a b}^{j}\right]$, there exists $C_{h_{j}} \in\left(\underline{Y}_{a b}^{j}, \bar{Y}_{a b}^{j}\right)$ such that $h_{j}^{\prime}\left(C_{h_{j}}\right)=$ $\left(h_{j}\left(\bar{Y}_{a b}^{j}\right)-h_{j}\left(\underline{Y}_{a b}^{j}\right)\right) /\left(\bar{Y}_{a b}^{j}-\underline{Y}_{a b}^{j}\right)$ by the mean value theorem.

Since $h_{j}^{\prime}\left(Y_{a b}^{j}\right)$ is monotonic function on $\left[\underline{Y}_{a b}^{j}, \bar{Y}_{a b}^{j}\right]$, there exists the inverse function of $h_{j}^{\prime}\left(Y_{a b}^{j}\right)$. Hence $C_{h_{j}}$ is uniquely given such that

$$
C_{h_{j}}=h_{j}^{\prime-1}\left(\frac{h_{j}\left(\bar{Y}_{a b}^{j}\right)-h_{j}\left(\underline{Y}_{a b}^{j}\right)}{\bar{Y}_{a b}^{j}-\underline{Y}_{a b}^{j}}\right) .
$$

When $\left\{h_{j}\right\}^{\prime \prime}>0$, we define the lower linear function $l_{h_{j}}$ as the following:

$$
\begin{aligned}
l_{h_{j}}\left(Y_{a b}^{j}\right):= & \frac{h_{j}\left(\bar{Y}_{a b}^{j}\right)-h_{j}\left(\underline{Y}_{a b}^{j}\right)}{\bar{Y}_{a b}^{j}-\underline{Y}_{a b}^{j}}\left(Y_{a b}^{j}-C_{h_{j}}\right) \\
& +h_{j}\left(C_{h_{j}}\right) .
\end{aligned}
$$

Similarly, $l_{h_{k j}}$ is defined as above.

Case $2\left(\left\{h_{j}\right\}^{\prime}<0\right)$. In the case, we put the valuable $Y_{a b}^{j} \in$ $\left[\underline{Y}_{a b}^{j}, \bar{Y}_{a b}^{j}\right]$; that is, $\underline{Y}_{a b}^{j}:=\min l_{b_{j}}^{u}(y) / \min l_{a_{j}}^{l}(y)$ and $\bar{Y}_{a b}^{j}:=$ $\max l_{b_{j}}^{u}(y) / \min l_{a_{j}}^{l}(y)$.

$$
\text { As }\left\{h_{j}\right\}^{\prime}<0, h_{j}\left(l_{b_{j}}^{u}(y) / l_{a_{j}}^{l}(y)\right) \leq h_{j}\left(b_{j}(y) / a_{j}(y)\right) \text {. }
$$

When $\left\{h_{j}\right\}^{\prime \prime}<0$, we define the linear functions $l_{h_{j}}$; that is,

$$
\begin{aligned}
l_{h_{j}}\left(Y_{a b}^{j}\right):= & \frac{h_{j}\left(\bar{Y}_{a b}^{j}\right)-h_{j}\left(\underline{Y}_{a b}^{j}\right)}{\bar{Y}_{a b}^{j}-\underline{Y}_{a b}^{j}}\left(Y_{a b}^{j}-\underline{Y}_{a b}^{j}\right) \\
& +h_{j}\left(\underline{Y}_{a b}^{j}\right) .
\end{aligned}
$$

When $\left\{h_{j}\right\}^{\prime \prime}>0$, we define $l_{h_{j}}$ as follows:

$$
\begin{aligned}
l_{h_{j}}\left(Y_{a b}^{j}\right):= & \frac{h_{j}\left(\bar{Y}_{a b}^{j}\right)-h_{j}\left(\underline{Y}_{a b}^{j}\right)}{\bar{Y}_{a b}^{j}-\underline{Y}_{a b}^{j}}\left(Y_{a b}^{j}-C_{h_{j}}\right) \\
& +h_{j}\left(C_{h_{j}}\right) .
\end{aligned}
$$

$l_{h_{k^{\prime}}}$ is also defined as the above. 
We have the lower bounded linearized optimization problem of $(P 0)$; that is,

$$
\begin{array}{ll}
\min & l_{f_{0}}(y)=\sum_{j=1}^{P} l_{h_{j}}(y) \\
\text { s.t. } & l_{f_{k}}(y)=\sum_{j=1}^{P_{k}} l_{h_{k j}}(y) \leq 0 .
\end{array}
$$

$\operatorname{LRP}(P 0)$

We rewrite our problem $(P)$ putting some technical assumption.

We assume $l_{a_{j}}^{l}(x)>0, l_{b_{j}}^{l}(x)>0, l_{c_{k^{\prime}}}^{l}(x)>0$, and $l_{d_{k^{\prime}}}^{l}(x)>$ 0 , and the problem is

$$
\begin{array}{ll}
\min & f_{0}(x)=\sum_{j=1}^{P} h_{j}\left(\frac{b_{j}(x)}{a_{j}(x)}\right) \\
\text { s.t. } & f_{k}(x)=\sum_{j=1}^{P_{k}} h_{k^{\prime} j}\left(\frac{d_{k^{\prime} j}(x)}{c_{k^{\prime} j}(x)}\right) \\
& \leq 0\left({ }^{\prime} j=1, \ldots, P_{k}, k=1, \ldots, M\right) \\
& x \in X,
\end{array}
$$

where $h_{j}$ on $\left[\min l_{b_{j}}^{l}(x) / \max l_{a_{j}}^{u}(x), \max l_{b_{j}}^{u}(x) / \min l_{a_{j}}^{l}(x)\right]$ and $h_{k^{\prime} j}$ on $\left[\min l_{d_{k^{\prime}}}^{l}(x) / \max l_{c_{k^{\prime}}}^{u}(x), \max l_{d_{k^{\prime}}}^{u}(x) / \min l_{c_{k^{\prime} j}}(x)\right]$ satisfied the following condition:

$$
\begin{array}{r}
h_{j}^{\prime}>0 \\
\text { or } h_{j}^{\prime}<0, \\
h_{k^{\prime} j}^{\prime}>0 \\
\text { or } h_{k^{\prime} j}^{\prime}<0, \\
\left\{h_{j}\right\}^{\prime \prime}>0 \\
\text { or }\left\{h_{j}\right\}^{\prime \prime}<0, \\
\left\{h_{k^{\prime}}\right\}^{\prime \prime}>0 \\
\text { or }\left\{h_{k^{\prime} j}\right\}^{\prime \prime}<0 .
\end{array}
$$

\section{Branch and Bound Algorithm}

In this section, we use the Simplex method and the Branch and Bound Algorithm and show how to find the approximate value of $(P 0)$.
We set the initial domain $Y^{0}:=Y$, the active domain set $\mathcal{Q}_{q}$, and the active domain $Y^{q(m)}=\left\{y \mid \underline{y}_{i}^{q(m)} \leq\right.$ $\left.y_{i}^{q(m)} \leq \bar{y}_{i}^{q(m)}, i=1, \ldots, N\right\} \subseteq Y^{0}$, where $q$ is the times of the cutting domains and the number of the stages in the algorithm and $m$ is the number of the active domains on stage $q$. If $Y^{q(m)}$ is active domain, we divide $Y^{q(m)}$ into half domains $Y^{q(m) \cdot 1}, Y^{q(m) \cdot 2}$. On each domain, we linearize the problem $(P 0)$ and solve the linearized problems $\operatorname{LRP}(P 0))$ to obtain the lower and upper bound values of $(P 0)$. After the repeat of the above calculations, we obtain the convergence for the sequences of the lower and upper bound values. The procedure leads the optimal value and the optimal solution for our problem.

3.1. Branching Rule. We select the branching variable $i$ such that $i=n \max \left\{\bar{y}_{n}^{q(m)}-y_{n}^{q(m)}, n=1,2, \ldots, N\right\}$. We divide the interval $\left[\underline{y}_{i}^{q(m)}, \bar{y}_{i}^{q(m)}\right]$ into half intervals: $\left[\underline{y}_{i}^{q(m)},\left(\underline{y}_{i}^{q(m)}+\right.\right.$ $\left.\left.\bar{y}_{i}^{q(m)}\right) / 2\right]$ and $\left[\left(\underline{y}_{i}^{q(m)}+\bar{y}_{i}^{q(m)}\right) / 2, \bar{y}_{i}^{q(m)}\right]$.

\subsection{Algorithm Statement}

Step 0 . Let $q$ be 0 , and let $m$ be 1 . We set an appropriate $\epsilon$-value as a convergence tolerance, the initial upper bound $V^{*}=\infty$, and $Q_{0}=\left\{Y^{0(1)}\right\}$. We solve $\operatorname{LRP}\left(Y^{0(1)}\right)$, and we write $\hat{y}\left(Y^{0(1)}\right)$ and $\mathrm{LB}_{0(1)}$ for the linear optimal solution and optimal value. If $\hat{y}\left(Y^{0(1)}\right)$ is feasible for $(P 0)$, update $V^{*}=f_{0}\left(\widehat{y}\left(Y^{0(1)}\right)\right)$ and we set the initial lower bound $\mathrm{LB}=\mathrm{LB}_{0(1)}$. If $V^{*}-\mathrm{LB} \leq \epsilon$, then we get the $\epsilon$-approximate optimal value $f_{0}\left(\widehat{y}\left(Y^{0(1)}\right)\right)$ and optimal solution $\hat{y}\left(Y^{0(1)}\right)$ of $(P 0)$, so we stop this algorithm. Otherwise, we proceed to Step 1.

Step 1. For all $m$, we divide $Y^{q(m)}$ into two half domains $Y^{q(m) \cdot 1}$ and $Y^{q(m) \cdot 2}$ according to above branching rule.

Step 2. For all $m$ and each domain $Y^{q(m) \cdot v}(v=1,2)$, we calculate

$$
\begin{aligned}
\underline{f}_{k(v)}= & \sum_{j=1, h_{k j}^{\prime}>0}^{P_{k}} h_{k^{\prime} j}\left(\underline{Y}_{c d}^{k^{\prime} j} \underline{Y}^{q(m) \cdot v}\right) \\
& +\sum_{j=1, h_{k j}^{\prime}<0}^{P_{k}} h_{k^{\prime} j}\left(\bar{Y}_{\overline{c d}}^{k^{\prime} j} Y^{q(m) \cdot v}\right) \quad(k=1, \ldots, M),
\end{aligned}
$$

where $\underline{Y}_{\underline{c d}}^{k^{\prime} Y^{q(m) \cdot v}}, \bar{Y}_{\overline{c d}}^{k^{\prime} Y^{q(m) \cdot v}}$ are defined on $Y^{q(m) \cdot v}$ :

$$
\begin{aligned}
& \underline{Y}^{k^{\prime} j} \underline{Y}^{q(m) \cdot v} \\
& :=\frac{\sum_{t=1, \beta_{k j t}^{d}>0}^{T_{k j}^{d}} \beta_{k j t}^{d} \exp \left(\sum_{i=1, \gamma_{k j t i}^{d}>0}^{N} \gamma_{k j t i}^{d} \underline{y}_{i}+\sum_{i=1, \gamma_{k j t i}^{d}<0}^{N} \gamma_{k j t i}^{d} \overline{y_{i}}\right)+\sum_{t=1, \beta_{k j t}^{d}<0}^{T_{k j}^{d}} \beta_{k j t}^{d} \exp \left(\sum_{i=1, \gamma_{k j t i}^{d}>0}^{N} \gamma_{k j t i}^{d} \overline{y_{i}}+\sum_{i=1, \gamma_{k j t i}^{d}<0}^{N} \gamma_{k j t i}^{d} y_{i}\right)}{\sum_{t=1, \beta_{k j t}^{c}>0}^{T_{k j}^{c}} \beta_{k j t}^{c} \exp \left(\sum_{i=1, \gamma_{k j t i}^{c}>0}^{N} \gamma_{k j t i}^{c} \overline{y_{i}}+\sum_{i=1, \gamma_{k j t i}^{c}<0}^{N} \gamma_{k j t i}^{c} y_{i}\right)+\sum_{t=1, \beta_{k j t}^{c}<0}^{T_{k j}^{c}} \beta_{k j t}^{c} \exp \left(\sum_{i=1, \gamma_{k j t i}^{c}>0}^{N} \gamma_{k j t i}^{c} y_{i}+\sum_{i=1, \gamma_{k j t i}^{c}<0}^{N} \gamma_{k j t i}^{c} \overline{y_{i}}\right)} .
\end{aligned}
$$




$$
\begin{aligned}
& \bar{Y}_{\overline{c d}}^{k^{\prime} Y^{q(m) \cdot v}} \\
& :=\frac{\sum_{t=1, \beta_{k j t}^{d}>0}^{T_{k j}^{d}} \beta_{k j t}^{d} \exp \left(\sum_{i=1, \gamma_{k j t i}^{d}>0}^{N} \gamma_{k j t i}^{d} \overline{y_{i}}+\sum_{i=1, \gamma_{k j t i}^{d}<0}^{N} \gamma_{k j t i}^{d} y_{i}\right)+\sum_{t=1, \beta_{k j t}^{d}<0}^{T_{k j}^{d}} \beta_{k j t}^{d} \exp \left(\sum_{i=1, \gamma_{k j t i}^{d}>0}^{N} \gamma_{k j t i}^{d} y_{i}+\sum_{i=1, \gamma_{k j t i}^{d}<0}^{N} \gamma_{k j t i}^{d} \overline{y_{i}}\right)}{\sum_{t=1, \beta_{k j t}^{c}>0}^{T_{k j}^{c}} \beta_{k j t}^{c} \exp \left(\sum_{i=1, \gamma_{k j t i}^{c}>0}^{N} \gamma_{k j t i}^{c} y_{i}+\sum_{i=1, \gamma_{k j t i}^{c}<0}^{N} \gamma_{k j t i}^{c} \overline{y_{i}}\right)+\sum_{t=1, \beta_{k j t}^{c}<0}^{T_{k j}^{c}} \beta_{k j t}^{c} \exp \left(\sum_{i=1, \gamma_{k j t i}^{c}>0}^{N} \gamma_{k j t i}^{c} \overline{y_{i}}+\sum_{i=1, \gamma_{k j t i}^{c}<0}^{N} \gamma_{k j t i}^{c} y_{i}\right)} .
\end{aligned}
$$

If there is $\underline{f}_{k(v)}$ that satisfies $\underline{f}_{k(v)}>0$ for some $k \in\{1,2, \ldots$, $M$ \}, the domain $Y^{q(m) \cdot v}$ is infeasible for $(P 0)$. In the case, we delete the domain from $Q_{q}$. If $Y^{q(m) \cdot v}(v=1,2)$ are deleted for all $m$, then the problem has no feasible solution.

Step 3. For left domains, we solve $\operatorname{LRP}\left(Y^{q(m) \cdot v}\right)$ by the Simplex algorithm, and we write $\left(\hat{y}\left(Y^{q(m) \cdot v}\right), \mathrm{LB}_{q(m) \cdot v}\right)$ for the obtained linear optimal solution and the value. If $\widehat{y}\left(Y^{q(m) \cdot v}\right)$ is feasible for $(P 0)$, we update $V^{*}=\min \left\{V^{*}, f_{0}\left(\widehat{y}\left(Y^{q(m) \cdot v}\right)\right)\right\}$. If $\mathrm{LB}_{q(m) \cdot v}>V^{*}$, we delete the corresponding domain from $Q_{q}$. If $V^{*}-\mathrm{LB}_{q(m) \cdot v} \leq \epsilon$, we obtain the $\epsilon$-approximate optimal value $f_{0}\left(\hat{y}\left(Y^{q(m) \cdot v}\right)\right)$ and optimal solution $\widehat{y}\left(Y^{q(m) \cdot v}\right)$ of $(P 0)$. Hence we stop this algorithm. Otherwise, we proceed to Step 4.

Step 4. We update the index of left domains $Y^{q(m) \cdot v}$ to $Y^{q+1(m)}$. We initialize $m$ and settle that $\widehat{Q}_{q+1}$ is a set of $Y^{q+1(m)}$ and go to Step 1.

\section{Convergence of the Optimization Method}

In this section, we prove the following two theorems to guarantee the convergence of our optimization method.

Theorem 1. If $|\bar{y}-y| \rightarrow 0$, then

$$
\begin{array}{r}
\left|\sum_{j=1}^{P} h_{j}(y)-\sum_{j=1}^{P} l_{h_{j}}(y)\right| \longrightarrow 0, \\
\left|\sum_{j=1}^{P_{k}} h_{k^{\prime}}(y)-\sum_{j=1}^{P_{k}} l_{h_{k j}}(y)\right| \longrightarrow 0 .
\end{array}
$$

One proves convergence (21). The convergence of (22) is proved by the same procedure as (21).

Proof. We show the following.

If $|\bar{y}-y| \rightarrow 0$, then $\left|h_{j}(y)-l_{h_{j}}(y)\right| \rightarrow 0$ for each $j$. Consider

$$
\begin{aligned}
& \left|h_{j}(y)-l_{h_{j}}(y)\right|=\mid h_{j}\left(\frac{b_{j}(y)}{a_{j}(y)}\right) \\
& \quad-l_{h_{j}}\left(\frac{l_{b_{j}}^{l}(y)}{\max l_{a_{j}}^{u}(y)}\right)|=| h_{j}\left(\frac{b_{j}(y)}{a_{j}(y)}\right)
\end{aligned}
$$

$$
\begin{aligned}
& -l_{h_{j}}\left(\frac{b_{j}(y)}{a_{j}(y)}\right)+l_{h_{j}}\left(\frac{b_{j}(y)}{a_{j}(y)}\right)-l_{h_{j}}\left(\frac{l_{b_{j}}^{l}(y)}{a_{j}(y)}\right) \\
& +l_{h_{j}}\left(\frac{l_{b_{j}}^{l}(y)}{a_{j}(y)}\right)-l_{h_{j}}\left(\frac{l_{b_{j}}^{l}(y)}{l_{a_{j}}^{u}(y)}\right)+l_{h_{j}}\left(\frac{l_{b_{j}}^{l}(y)}{l_{a_{j}}^{u}(y)}\right) \\
& -l_{h_{j}}\left(\frac{l_{b_{j}}^{l}(y)}{\max l_{a_{j}}^{u}(y)}\right)|\leq| h_{j}\left(\frac{b_{j}(y)}{a_{j}(y)}\right) \\
& -l_{h_{j}}\left(\frac{b_{j}(y)}{a_{j}(y)}\right)|+| l_{h_{j}}\left(\frac{b_{j}(y)}{a_{j}(y)}\right)-l_{h_{j}}\left(\frac{l_{b_{j}}^{l}(y)}{a_{j}(y)}\right) \mid \\
& +\left|l_{h_{j}}\left(\frac{l_{b_{j}}^{l}(y)}{a_{j}(y)}\right)-l_{h_{j}}\left(\frac{l_{b_{j}}^{l}(y)}{l_{a_{j}}^{u}(y)}\right)\right|+\mid l_{h_{j}}\left(\frac{l_{b_{j}}^{l}(y)}{l_{a_{j}}^{u}(y)}\right) \\
& -l_{h_{j}}\left(\frac{l_{b_{j}}^{l}(y)}{\max l_{a_{j}}^{u}(y)}\right) \mid \cdot
\end{aligned}
$$

We prove the convergence of the 4 terms of the above.

(i) The proof of $\left|h_{j}\left(b_{j}(y) / a_{j}(y)\right)-l_{h_{j}}\left(b_{j}(y) / a_{j}(y)\right)\right| \rightarrow 0$ is as follows.

When $h_{j}^{\prime \prime}>0$, we define the lower linearized function of $h_{j}(y)$; that is,

$$
\begin{aligned}
l_{h_{j}}\left(Y_{a b}^{j}\right):= & \frac{h_{j}\left(\bar{Y}_{a b}^{j}\right)-h_{j}\left(\underline{Y}_{a b}^{j}\right)}{\bar{Y}_{a b}^{j}-\underline{Y}_{a b}^{j}}\left(Y_{a b}^{j}-C_{h_{j}}\right) \\
& +h_{j}\left(C_{h_{j}}\right) .
\end{aligned}
$$

Then

$$
\begin{aligned}
& \max \left|h_{j}\left(Y_{a b}^{j}\right)-l_{h_{j}}\left(Y_{a b}^{j}\right)\right|=\mid h_{j}\left(Y_{a b}^{j}\right) \\
& -\left(\frac{h_{j}\left(\bar{Y}_{a b}^{j}\right)-h_{j}\left(\underline{Y}_{a b}^{j}\right)}{\bar{Y}_{a b}^{j}-\underline{Y}_{a b}^{j}}\left(Y_{a b}^{j}-C_{h_{j}}\right)\right. \\
& \left.+h_{j}\left(C_{h_{j}}\right)\right) \mid .
\end{aligned}
$$


We put $I_{Y_{a b}^{j}}:=\bar{Y}_{a b}^{j}-\underline{Y}_{a b}^{j}, C_{h_{j}}=\underline{Y}_{a b}^{j}+\theta_{a b}^{j} \cdot I_{Y_{a b}^{j}}\left(0 \leq \theta_{a b}^{j} \leq 1\right)$. It is

$$
\begin{aligned}
& \mid h_{j}\left(Y_{a b}^{j}\right) \\
& \quad-\frac{h_{j}\left(\bar{Y}_{a b}^{j}\right)-h_{j}\left(\underline{Y}_{a b}^{j}\right)}{\bar{Y}_{a b}^{j}-\underline{Y}_{a b}^{j}}\left(Y_{a b}^{j}-\underline{Y}_{a b}^{j}-\theta_{a b}^{j} \cdot I_{Y_{a b}^{j}}\right) \\
& -h_{j}\left(\underline{Y}_{a b}^{j}+\theta_{a b}^{j} \cdot I_{Y_{a b}^{j}}\right)|=| h_{j}\left(\underline{Y}_{a b}^{j}\right) \\
& \quad+\left(h_{j}\left(\bar{Y}_{a b}^{j}\right)-h_{j}\left(\underline{Y}_{a b}^{j}\right)\right) \theta_{a b}^{j} \cdot I_{Y_{a b}^{j}} \\
& -h_{j}\left(\underline{Y}_{a b}^{j}+\theta_{a b}^{j} \cdot I_{Y_{a b}^{j}}\right) \mid \cdots .
\end{aligned}
$$

If $|\bar{y}-\underline{y}| \rightarrow 0$, then $\theta_{a b}^{j} \cdot I_{Y_{a b}^{j}} \rightarrow 0$. Hence $(* 1) \rightarrow 0$.

When $h_{j}^{\prime \prime}<0$, we define $l_{h_{j}}\left(Y_{a b}^{j}\right)$ as follows:

$$
\begin{aligned}
& l_{h_{j}}\left(Y_{a b}^{j}\right):=\frac{h_{j}\left(\bar{Y}_{a b}^{j}\right)-h_{j}\left(\underline{Y}_{a b}^{j}\right)}{\bar{Y}_{a b}^{j}-\underline{Y}_{a b}^{j}}\left(Y_{a b}^{j}-\underline{Y}_{a b}^{j}\right) \\
& +h_{j}\left(\underline{Y}_{a b}^{j}\right) . \\
& \max \left|h_{j}\left(Y_{a b}^{j}\right)-l_{h_{j}}\left(Y_{a b}^{j}\right)\right|=\left|h_{j}\left(C_{h_{j}}\right)-l_{h_{j}}\left(C_{h_{j}}\right)\right| \\
& =\mid h_{j}\left(\underline{Y}_{a b}^{j}+\theta_{a b}^{j} \cdot I_{Y_{a b}^{j}}\right) \\
& \quad-\left(\frac{h_{j}\left(\bar{Y}_{a b}^{j}\right)-h_{j}\left(\underline{Y}_{a b}^{j}\right)}{\bar{Y}_{a b}^{j}-\underline{Y}_{a b}^{j}}\left(\underline{Y}_{a b}^{j}+\theta_{a b}^{j} \cdot I_{Y_{a b}^{j}}-\underline{Y}_{a b}^{j}\right)\right. \\
& \left.-h_{j}\left(\underline{Y}_{a b}^{j}\right)\right) \mid \ldots .
\end{aligned}
$$

When $|\bar{y}-\underline{y}| \rightarrow 0$, then $\theta_{a b}^{j} \cdot I_{Y_{a b}^{j}} \rightarrow 0$. Then $(* 2) \rightarrow 0$.

(ii) The proof of $\left|l_{h_{j}}\left(b_{j}(y) / a_{j}(y)\right)-l_{h_{j}}\left(l_{b_{j}}^{l}(y) / a_{j}(y)\right)\right| \rightarrow 0$ is as follows.

We show that $\left|b_{j}(y)-l_{b_{j}}^{l}(y)\right| \rightarrow 0$ if $|\bar{y}-\underline{y}| \rightarrow 0$.

By the definition of $L_{\beta_{j t}^{b}}^{l}\left(Y_{j t}^{b}\right)$, we show that $\mid \beta_{j t}^{b} \exp \left(Y_{j t}^{b}\right)-$ $L_{\beta_{j t}^{b}}^{l}\left(Y_{j t}^{b}\right) \mid \rightarrow 0$ for any $t$ similarly to (i).

(iii) The proof of $\left|l_{h_{j}}\left(l_{b_{j}}^{l}(y) / a_{j}(y)\right)-l_{h_{j}}\left(l_{b_{j}}^{l}(y) / l_{a_{j}}^{u}(y)\right)\right| \rightarrow 0$ is as follows.

We prove that $\left|a_{j}(y)-l_{a_{j}}^{u}(y)\right| \rightarrow 0$ if $|\bar{y}-\underline{y}| \rightarrow 0$.
By the definition of $L_{\beta_{j t}^{a}}^{u}\left(Y_{j t}^{a}\right)$, we show that $\mid \beta_{j t}^{a} \exp \left(Y_{j t}^{a}\right)-$ $L_{\beta_{j t}^{a}}^{u}\left(Y_{j t}^{a}\right) \mid \rightarrow 0$ for any $t$ similarly to (i).

(iv) The proof of $\left|l_{h_{j}}\left(l_{b_{j}}^{l}(y) / l_{a_{j}}^{u}(y)\right)-l_{h_{j}}\left(l_{b_{j}}^{l}(y) / \max l_{a_{j}}^{u}(y)\right)\right|$ $\rightarrow 0$ is as follows.

If $|\bar{y}-\underline{y}| \rightarrow 0$, then $l_{a_{j}}^{u}(y) \rightarrow \max l_{a_{j}}^{u}(y)$.

Theorem 2. Suppose that problem (P0) has a global optimal solution, denoted by $\mu_{0}^{*}$. Then one has the following.

(i) For the case $\epsilon>0$ : the algorithm always terminates after finitely many iterations yielding a global $\epsilon$-optimal solution $y^{*}$ and a global $\epsilon$-optimal value $V^{*}$ for problem $(P 0)$ in the sense that $y^{*} \in Y, V^{*}-\epsilon \leq \mu_{0}^{*}$ with $V^{*}=f_{0}\left(y^{*}\right)$.

(ii) For the case $\epsilon \rightarrow 0$ : one assumes the sequence $\epsilon_{n}$ is convergence tolerance, such that $\epsilon_{1}>\epsilon_{2}>\cdots>\epsilon_{n}>\epsilon_{n+1}>$ $\cdots>0$; that is, $\lim _{n \rightarrow \infty} \epsilon_{n}=0$. And we assume the sequence $y_{n}^{*}$ is optimal solution of $(P 0)$ corresponding to $\epsilon_{n}$. Then the accumulation point of $y_{n}^{*}$ is global optimal solution of $(P 0)$.

Proof. (i) It is obvious by the algorithm statement. is $V_{n}^{*}$ :

(ii) We denote that the upper bound corresponding to $\epsilon_{n}$

$$
f_{0}\left(y_{n}^{*}\right) \in\left[V_{n}^{*}-\epsilon, V_{n}^{*}\right] .
$$

Then $\left\{y_{n}^{*}\right\}$ is the point sequence on bounded closed set, and $\left\{y_{n}^{*}\right\}$ has a converge subsequence $\left\{y_{n i}^{*}\right\}$.

We denote $\lim _{i \rightarrow \infty} y_{n i}^{*}=y^{*}$, and then

$$
\begin{aligned}
V_{n i}^{*}-\epsilon_{n i} & \leq f_{0}\left(V_{n i}^{*}\right) \leq V_{n i}^{*} . \\
\text { If } i & \longrightarrow \infty, \\
\text { then } n i & \longrightarrow \infty, \\
\lim _{n i \rightarrow \infty} \epsilon_{n i} & =0 .
\end{aligned}
$$

Now $\left\{V_{n}^{*}\right\}$ is a monotone decreasing sequence; therefore it is convergent. We put $\lim _{n \rightarrow \infty} V_{n}^{*}=\mu_{0}^{*}$ :

$$
\lim _{i \rightarrow \infty}\left(V_{n i}^{*}-\epsilon_{n i}\right) \leq \lim _{i \rightarrow \infty} f_{0}\left(y_{n i}^{*}\right) \leq \lim _{i \rightarrow \infty} V_{n i}^{*} .
$$

Since $f_{0}(y)$ is a continuous function, $\lim _{i \rightarrow \infty} f_{0}\left(y_{n i}\right)=$ $f_{0}\left(y^{*}\right)$. Therefore, $\mu_{0}^{*} \leq f_{0}\left(y^{*}\right) \leq \mu_{0}^{*}$, and $f_{0}\left(y^{*}\right)=\mu_{0}^{*}$. Since $f_{k}\left(y_{n}^{*}\right) \leq 0$, for each $k$, and $f_{k}\left(y^{*}\right)$ is continuous, we obtain that $\lim _{n \rightarrow \infty} f_{k}\left(y_{n}^{*}\right)=f_{k}\left(y^{*}\right) \leq 0$.

\section{Numerical Experiment}

In this section, we show some numerical experiments for these optimization problems according to the former rules. We make the algorithm coded with Matlab. In these codes, we use Matlab's unique function code "linprog" to solve the linear optimization problems. 
Example 1. Consider

$$
\begin{array}{ll}
\min & \left(\exp \left(\frac{-x_{1}^{2}+3 x_{1}+2 x_{2}^{2}+3 x_{2}+3.5}{x_{1}+1}\right)-\exp \left(\frac{x_{2}}{x_{1}^{2}-2 x_{1}+x_{2}^{2}-8 x_{2}+20}\right)\right) \\
\text { s.t. } & x_{1}-\frac{x_{2}}{x_{1}} \leq 1 \\
& 2 \frac{x_{1}}{x_{2}}+x_{2} \leq 6 \\
& X=\left\{x: 1 \leq x_{1} \leq 3,1 \leq x_{2} \leq 3\right\} .
\end{array}
$$

We set $\epsilon=0.000001$. After the algorithm, we found a global $\epsilon$-optimal value $V^{*}=59.3054$ when the global $\epsilon$-optimal solution is $\left(x_{1}, x_{2}\right)^{T}=(1,1.6180)$.

$$
\begin{array}{ll}
\min & \left(\sin \left(\frac{x_{1}^{2}+2 x_{2}-2 x_{1}+x_{2}^{2}}{x_{1}+x_{2}^{2}+4}\right)+\cos \left(\frac{3 x_{1}^{2}-3 x_{2}+2 x_{1}+x_{2}^{2}+3}{x_{1}^{2}+2 x_{2}^{2}+10}\right)\right) \\
\text { s.t. } & \sin \left(\frac{x_{1}^{2}+3 x_{2}-2 x_{2}^{2}+2}{x_{1}^{2}+x_{2}+5}\right)+\cos \left(\frac{-x_{2}^{2}+2 x_{1}+2 x_{2}}{x_{1}+5}\right) \leq 0 \\
& X=\left\{x: 1 \leq x_{1} \leq 2,1 \leq x_{2} \leq 2\right\} .
\end{array}
$$

We set $\epsilon=0.000001$. After the algorithm, we found a global $\epsilon$-optimal value $V^{*}=0.9023$ when the global $\epsilon$-optimal solution is $\left(x_{1}, x_{2}\right)^{T}=(2,1)$.

\section{Example 3. Consider}

$$
\begin{array}{ll}
\min & \left(\log \left(\frac{2 x_{1}^{2}-x_{2}+35}{-x_{1}+2 x_{2}^{2}+9}\right)-\log \left(\frac{3 x_{1}^{2}-x_{2}+35}{x_{1}^{2}-x_{1}+x_{2}^{2}+2 x_{2}+3}\right)\right) \\
\text { s.t. } & x_{1}^{2}-2 x_{2} \leq 1 \\
& x_{1}-\frac{x_{2}}{x_{1}} \leq 1 \\
& X=\left\{x: 1 \leq x_{1} \leq 3,1 \leq x_{2} \leq 3\right\} .
\end{array}
$$

We set $\epsilon=0.000001$. After the algorithm, we found a global $\epsilon$-optimal value $V^{*}=-0.5382$ when the global $\epsilon$-optimal solution is $\left(x_{1}, x_{2}\right)^{T}=(1,1)$.

\section{Concluding Remarks}

In this paper, we propose the specific nonlinear and nonconvex optimization technique which does not launch new valuables applying Hongwei's method [6]. We compute the examples of our previous work [17] by the new method. In [17], it had taken over 8 hours to find optimal value for each problem. The proposed method can compute the same problems in 10 minutes. If the algorithms are coded by $\mathrm{C}$ or $\mathrm{C}++$, we obtain the optimal value in a shorter time.

\section{Competing Interests}

The authors declare that there are no competing interests regarding the publication of this paper.

\section{Acknowledgments}

The authors would like to thank Y. Yamamoto for arranging the system of computers. 


\section{References}

[1] P. Shen, Y. Duan, and Y. Ma, "A robust solution approach for nonconvex quadratic programs with additional multiplicative constraints," Applied Mathematics and Computation, vol. 201, no. 1-2, pp. 514-526, 2008.

[2] C. A. Floudas and C. E. Gounaris, "A review of recent advances in global optimization," Journal of Global Optimization, vol. 45, no. 1, pp. 3-38, 2009.

[3] Y. Ji, K.-C. Zhang, and S.-J. Qu, "A deterministic global optimization algorithm," Applied Mathematics and Computation, vol. 185, no. 1, pp. 382-387, 2007.

[4] H. Jiao, Z. Wang, and Y. Chen, "Global optimization algorithm for sum of generalized polynomial ratios problem," Applied Mathematical Modelling, vol. 37, no. 1-2, pp. 187-197, 2013.

[5] S. Pei-Ping and Y. Gui-Xia, "Global optimization for the sum of generalized polynomial fractional functions," Mathematical Methods of Operations Research, vol. 65, no. 3, pp. 445-459, 2007.

[6] M. Chiang, "Nonconvex optimization for communication networks," in Advances in Applied Mathematics and Global Optimization, vol. 17 of Applied Mathematics and Mechanics, pp. 137196, Springer, New York, NY, USA, 2009.

[7] J.-W. Lee, R. R. Mazumdar, and N. B. Shroff, "Non-convex optimization and rate control for multi-class services in the internet," IEEE/ACM Transactions on Networking, vol. 13, no. 4, pp. 827-840, 2005.

[8] D. Cai and T. G. Nitta, "Limit of the solutions for the finite horizon problems as the optimal solution to the infinite horizon optimization problems," Journal of Difference Equations and Applications, vol. 17, no. 3, pp. 359-373, 2011.

[9] D. Cai and T. G. Nitta, "Optimal solutions to the infinite horizon problems: constructing the optimum as the limit of the solutions for the finite horizon problems," Nonlinear Analysis: Theory, Methods \& Applications, vol. 71, no. 12, pp. e2103-e2108, 2009.

[10] R. Okumura, D. Cai, and T. G. Nitta, "Transversality conditions for infinite horizon optimality: higher order differential problems," Nonlinear Analysis: Theory, Methods \& Applications, vol. 71, no. 12, pp. e1980-e1984, 2009.

[11] H. Konno and H. Watanabe, "Bond portfolio optimization problems and their applications to index tracking: a partial optimization approach," Journal of the Operations Research Society of Japan, vol. 39, no. 3, pp. 295-306, 1996.

[12] H. P. Benson, "Using concave envelopes to globally solve the nonlinear sum of ratios problem," Journal of Global Optimization, vol. 22, no. 1, pp. 343-364, 2002.

[13] H. Konno and K. Fukaishi, "A branch and bound algorithm for solving low rank linear multiplicative and fractional programming problems," Journal of Global Optimization, vol. 18, no. 3, pp. 283-299, 2000.

[14] N. T. H. Phuong and H. Tuy, "A unified monotonic approach to generalized linear fractional programming," Journal of Global Optimization, vol. 26, no. 3, pp. 229-259, 2003.

[15] M. Horai, H. Kobayashi, and T. G. Nitta, "Global optimization for the sum of certain nonlinear functions," Abstract and Applied Analysis, vol. 2014, Article ID 408918, 8 pages, 2014.

[16] H. Jiao and Y. Chen, "A note on a deterministic global optimization algorithm," Applied Mathematics and Computation, vol. 202, no. 1, pp. 67-70, 2008.
[17] Y. Gao, Y. Shang, and L. Zhang, "A branch and reduce approach for solving nonconvex quadratic programming problems with quadratic constraints," OR Transactions, vol. 9, no. 2, pp. 9-20, 2005.

[18] J. Linderoth, "A simplicial branch-and-bound algorithm for solving quadratically constrained quadratic programs," Mathematical Programming, vol. 103, no. 2, pp. 251-282, 2005. 


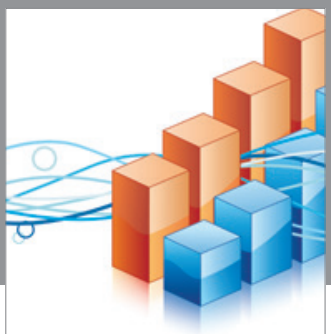

Advances in

Operations Research

vatem alat4

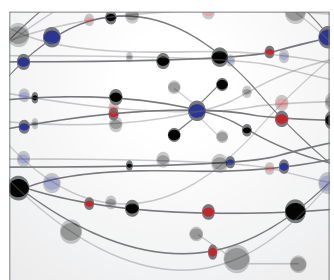

\section{The Scientific} World Journal
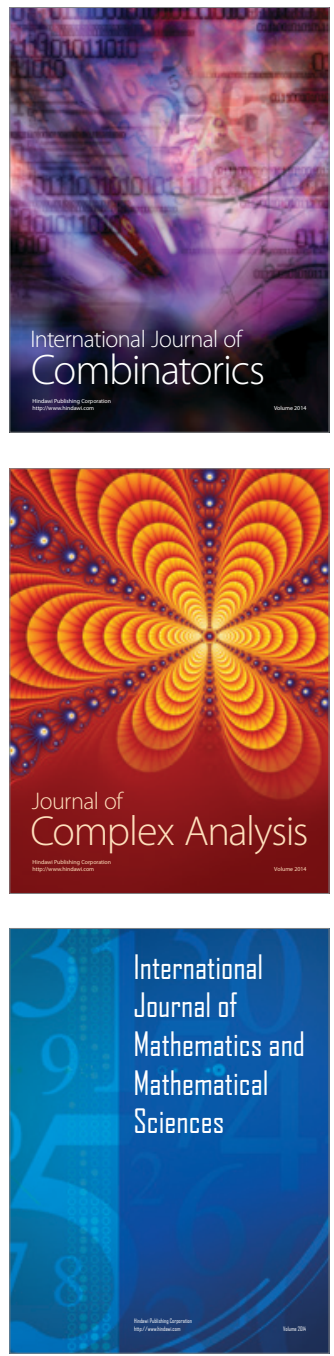
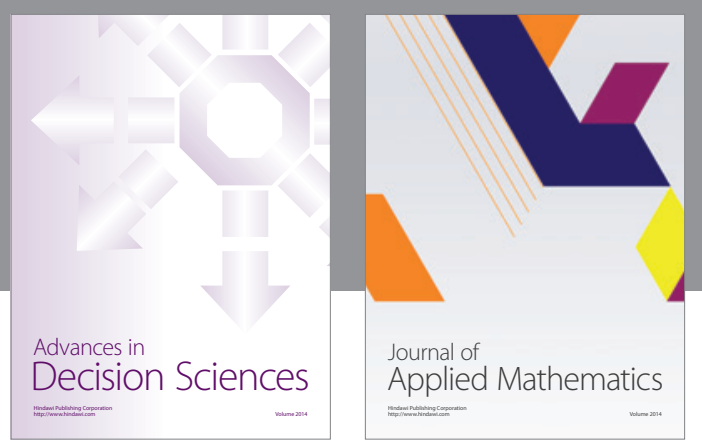

Algebra

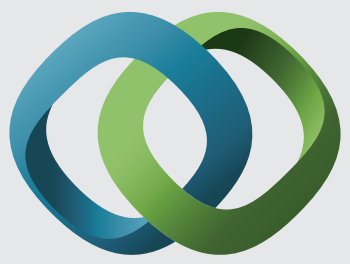

\section{Hindawi}

Submit your manuscripts at

http://www.hindawi.com
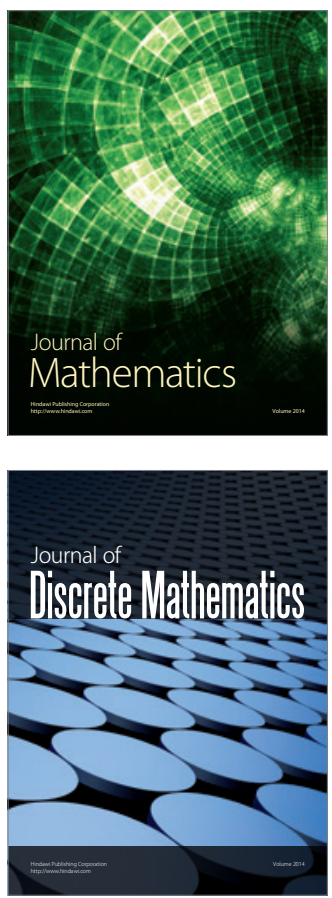

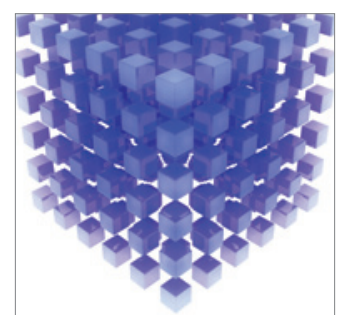

Mathematical Problems in Engineering
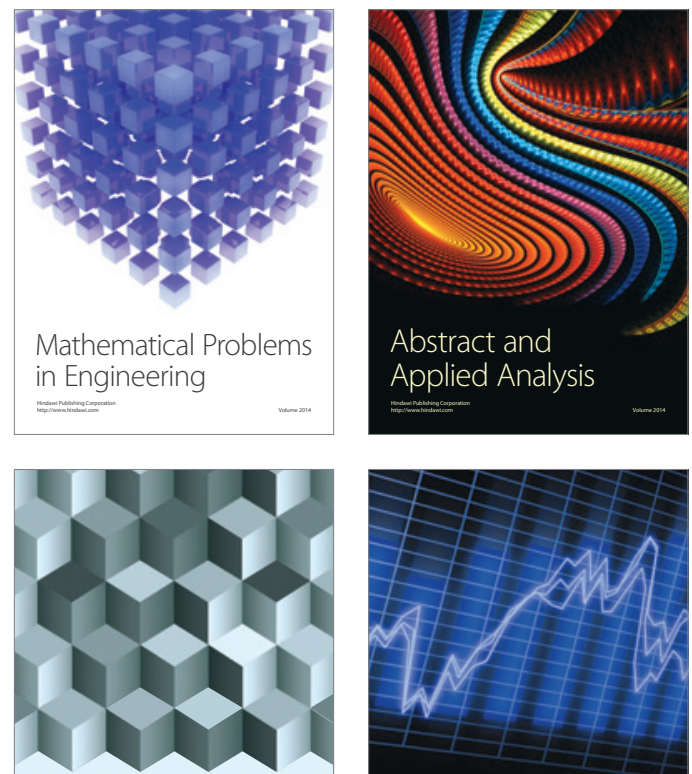

Journal of

Function Spaces

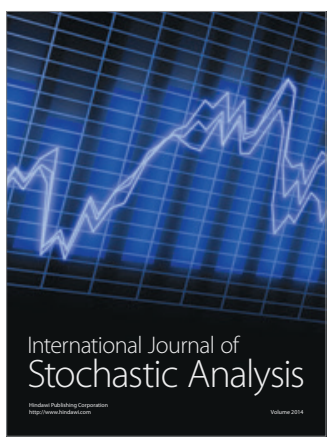

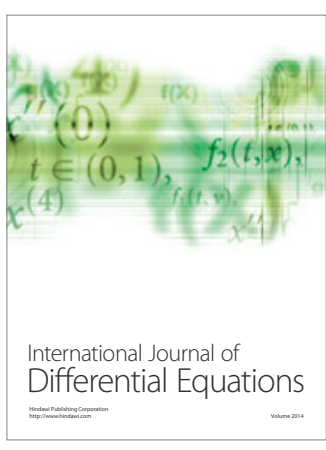
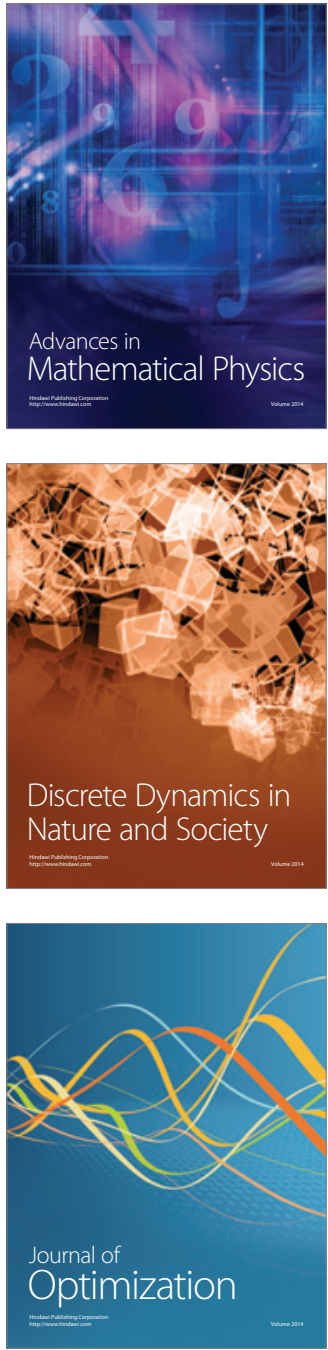